\title{
Analytical comparison for square, rectangular and circular diaphragms in MEMS applications
}

\begin{abstract}
MEMS offer different fabrication process to fabricate diaphragms in various applications such as microphones, pressure sensors and micro-pumps, etc. The working principle of all these devices is based on the force applies on the one side of the diaphragm and cause the diaphragm deflects. The parameter optimization of diaphragm is the prime challenge due to achieve the best performance of the device. In this paper Displacement, Stress and Vibration analysis which affect on the operation of the diaphragm under pressures is considered. The different shapes of diaphragms (square, rectangular and circular shapes) are also play a significant role in operation in different applications. The purpose of this research is to design a framework to show which kind of diaphragm with what kind of parameters can be chosen for specific MEMS devices.
\end{abstract}

Keyword: MEMS; Diaphragm; Stress 